\title{
INFECTION ET MALNUTRITION CHEZ LES ENFANTS ET LES FEMMES EN AGE DE PROCREER AU MALI.
}

\section{INFECTION AND MALNUTRITION AMONG THE CHILDREN AND WOMEN IN AGE OF PROCREER IN MALI.}

AG IKNANE $A^{1}$, DIAWARA $A^{2}$, DJANGO DM ${ }^{3}$, THIERO TA ${ }^{4}$, LANDOURE $A^{5}$, NIANGALY ${ }^{6}$, FOFANA $A^{7}$, KAMIAN K$^{8}$, CISSE AT ${ }^{9}$, TRAORE A K ${ }^{10}$ 1 Maître assistant en santé publique à la FMPOS, Chef du service Nutrition à l'Institut
National de Recherche en Santé Publique - Bamako, Mali
${ }^{2}$ Maître assistant en santé publique à la FMPOS, Directeur de l'Agence Nationale
d'Evaluation des Hôpitaux (ANEH), Bamako - Mali
${ }^{3}$ Maître assistant en anesthésie réanimation, CHU Gabriel Touré, Bamako
${ }^{4}$ Biologiste, administrateur des services de santé à l'Institut National de Recherche
en Santé Publique, Bamako
5 Epidémiologiste à l'Institut National de Recherche en Santé Publique (INRSP),
Bamako
${ }^{6}$ Maître de recherche en santé communautaire à l'Institut National de Recherche en Santé Publique, Bamako

${ }^{7}$ Agronome, en service à la Division du Suivi de la Situation alimentaire et nutritionnelle (DSSAN) du Ministère de la santé, Bamako

8 Pharmacienne Biochimiste à l'Institut National de Recherche en Santé Publique (INRSP), Bamako

${ }_{9}^{9}$ Nutritionniste, responsable du projet Doumini Nafama, Hellen keller International, Bamako

10 Maître de conférences agrégé en médecine interne, Directeur du Centre National d'Appui à la Lutte contre la maladie (CNAM) - Bamako

Remerciements: Nous remercions Heller Keller International pour le financement de la présente etude.

Résumé

La malnutrition et les infections sont des problèmes communs de santé publique des enfants dans les pays en voie de développement. Dans le but de déterminer la prévalence de la sous-nutrition et de l'infection et de leurs liens possibles, une étude transversale stratifiée par sondage en grappe a été réalisée du 30 janvier au 16 mars 2006 dans deux régions du Mali : Koulikoro (zone rurale) et le district de Bamako (zone urbaine). Elle a porté sur un échantillon de 692 enfants de 24 à 59 mois et 803 femmes en âge de procréer. Les mesures anthropométriques et le dosage de la $\mathrm{C}$ réactive Protéine (CRP) avaient été réalisés pour déterminer la prévalence de la sous-nutrition.

La prévalence de l'infection chez les enfants étudiés était significativement plus accentuée en milieu urbain avec $35,2 \pm 0,47 \%$ qu'en milieu rural avec 14,8 $\pm 0,35 \%$ $(p=0,0001)$. Chez les femmes en âge de procréer par contre, elle était comparable dans les deux milieux avec $24,8 \pm 0,35 \%$ en milieu rural et $17,6 \pm 0,47 \%$ en milieu urbain. $(p<0,05)$. Les enfants citadins sont statistiquement plus infectés que ceux du milieu rural $(p=0,000)$ avec respectivement $35,2 \%$ contre $14,8 \%$. Quant aux femmes, c'est plutôt l'inverse avec 4,8\% contre $17,6 \%$ bien que cela ne soit pas significatif $(p<0,05)$. La proportion des enfants émaciés et infectés est comparable dans les deux milieux avec respectivement $6,1 \%$ en milieu rural et $4,6 \%$ en milieu urbain. Par contre en milieu rural, la proportion d'enfants ayant un retard de croissance et une insuffisance pondérale étaient significativement plus infectées que ceux du milieu urbain $(p=0,000)$. La relation entre l'insuffisance pondérale et les autres carences nutritionnelles chez les enfants de 24 à 59 mois est statistiquement significative avec le retard de croissance et l'émaciation dans les deux milieux, mais la force de l'association pour l'émaciation n'est pas très forte en milieu urbain $(p=0,004)$. Chez les femmes, aucune relation significative n'avait été observée entre infection et déficit énergétique chronique.

Mots clés: Infection, Déficit énergétique chronique, émaciation, retard de croissance, insuffisance pondérale.

\section{Summary}

Malnutrition and the infections are common problems of public health of the children in the countries in the process of development. With an aim of determining the prévalence of the malnutrition and infection and their possible relations, a cross-sectional study laminated by cluster sampling was carried out of January 30 at March 16, 2006 in two areas of Mali: Koulikoro (rural rural) and the district of Bamako (urban zone). It related to a sample of 692 children from 24 to 59 months and 803 women in age to procreate. Anthropometric measurements and the proportioning of $\mathrm{C}$ reactive Proteine (CRP) had been carried out to determine the prevalency of the malnutrition

The prevalence of the infection in the studied children significantly was accentuated in urban environment with $35.2 \pm 0.47 \%$ than in rural medium with $14.8 \pm 0.35 \%$ $(p=0.0001)$. Among women in age to procreate on the other hand, it was comparable in the two mediums with $24.8 \pm 0.35 \%$ in rural medium and $17.6 \pm 0.47 \%$ in urban environment $(p<0.05)$. The urban children are infected statistically than those of the rural medium $(p=0,000)$ with respectively $35.2 \%$ against $14.8 \%$. As for the women, it is rather the reverse with $4.8 \%$ against $17.6 \%$ although that is not significant $(p<0.05)$. The proportion of the wasting and infected children is comparable in the two mediums with respectively $6.1 \%$ in rural medium and $4.6 \%$ in urban environment. On the other hand in rural medium, the proportion of children having a statung and a underweight significantly were infected than those of the urban environment $(p=0.000)$. The relation between underground and the other nutritional deficiencies in the children from 24 to 59 months is statistically significant with the delay of growth and the wasting in the two mediums, but the force of association for the wasting is not very strong in urban environment $(p=0.004)$. Among women, no significant relation had been observed between infection and chronic energy deficit.

Keys words: Infection, Chronic energy deficient, wasting , stunting, Underground. 


\section{Introduction}

Dans le monde, la malnutrition touche une personne sur trois et chacune de ses formes principales éclipse la plupart des autres maladies à l'échelle mondiale. La malnutrition affecte tous les groupes d'âge, mais elle est particulièrement fréquente chez les pauvres et ceux qui ont un accès insuffisant à l'eau propre et à un assainissement de bonne qualité et sont privés d'éducation sanitaire. Plus de $70 \%$ des enfants souffrant de malnutrition protéino-énergétique vivent en Asie, $26 \%$ vivent en Afrique et $4 \%$ en Amérique latine et dans les Caraïbes [1].

Globalement, environ 60 millions d'enfants souffrent de malnutrition aiguë modérée et 13 millions souffrent de la forme sévère. Environ $9 \%$ des enfants en Afrique subSaharienne et $15 \%$ des enfants en Asie du sud souffrent de malnutrition aigue modérée, et environ $2 \%$ des enfants dans les pays en développement souffrent de malnutrition aigue sévère [2].

Les pénuries alimentaires chroniques touchent environ 792 millions de personnes dans le monde [3], soit près de $20 \%$ de la population des pays en développement. Dans les pays en développement (PED), pauvreté, malnutrition et insuffisance des services de santé sont à l'origine chaque année du décès de millions de personnes dont une majorité d'enfants qui meurent dénutris, victimes de l'interaction entre la malnutrition et cinq ou six maladies évitables. Outre la malnutrition protéino-énergétique, les carences spécifiques en micronutriments ont également un impact sur l'immunité et entraînent un risque accru de mortalité par infection [4]. Face aux agents pathogènes, le système immunitaire, capable de détecter et d'éliminer les intrus ayant franchi les premières lignes de défense, présente un fonctionnement fortement conditionné par l'état nutritionnel. L'immunodéficience acquise secondaire à la malnutrition entraîne une chute du potentiel de défense et donc une moindre résistance aux infections [4]. Lors de l'installation d'une infection, divers processus biochimiques, métaboliques et hormonaux produisent à leur tour un effet délétère sur l'état nutritionnel en modifiant l'équilibre entre apports et besoins. L'organisme entre alors dans une série de cercles vicieux, véritable spirale morbide expliquant le taux élevé de mortalité liée au couple "malnutrition-infection".

La présente étude s'inscrivait dans le cadre d'une étude de base en vue d'instaurer un programme de nutrition visant la fortification d'aliments de grande consommation. Son objectif est d'évaluer le statut nutritionnel et le niveau d'infection des femmes en âge de procréer et des enfants de 24 à 59 mois dans le milieu urbain du district de Bamako et en milieu rural de la région de Koulikoro à travers les mesures anthropométriques et le dosage de la $C$ réactive Protéine et déterminer le lien entre infection et malnutrition.

\section{Matériels et méthodes}

L'étude était de type transversal stratifié avec un sondage en grappe à trois degré à l'intérieur de chaque strate et s'est déroulée 30 janvier au 16 mars 2006. Le cadre de l'étude était constituée par une zone urbaine comprenant les 6 communes du district de Bamako pour une population de 1.016167 habitants [5] et une zone rurale constituée par les communes rurales des cercles de Koulikoro (4 communes), Kolokani (4 communes), Kati (9 communes), Kangaba (2 communes), Banamba (3 communes), Doïla (5 communes) et Nara (4 communes) avec une population totalisant 1516486 habitants. La population d'étude était constituée par les enfants de 24 à 59 mois (440 en zone rurale de Koulikoro et 363 en zone urbaine de Bamako) et les femmes en âge de procréer (445 à Koulikoro et 247 à Bamako).

Le nombre de ménages retenu dans l'étude a été calculé à partir de la formule habituelle (nombre de ménages = Nombre de femmes) / (taux de réponse * nombre de femmes par ménage). Un taux de réponse de $90 \%$ utilisé par I'EDSM III [6] a été retenu. La taille de l'échantillon a été déterminée par la formule usuelle de Schwartz [3]. Des prélèvements de sang veineux chez les femmes et les enfants ont été réalisés pour le dosage de la C-Réactive Protéine (CRP).

Le degré d'infection a été évalué grâce au CRP [1], qui est une protéine présente en faible quantité dans le sérum normal. Dans les processus inflammatoires (infection bactérienne liée à une lésion tissulaire ou consécutive à une opération chirurgicale) sa concentration est augmentée de manière significative, marqueur précoce de l'infection bactérienne [1]. Le Quick Read CRP utilise une méthode immunoturbidimétrique basée sur la réaction entre un fragment anti humain CRP $\mathrm{F}(\mathrm{ab})_{2}$ et le CRP présent dans le sérum. II en résulte une turbidité dont on mesure l'absorbance. Les valeurs Prédictives sont définies avec une valeur normale $<5$ $\mathrm{mg} / \mathrm{L}$; une valeur comprise entre 5 et $10 \mathrm{mg} / \mathrm{L}$ peut signifier un faible processus inflammatoire, entre 10 et 50 $\mathrm{mg} / \mathrm{L}$ il s'agissait d'un processus inflammatoire modéré dû à une infection aiguë et au delà $50 \mathrm{mg} / \mathrm{l}$ il s'agira d'activité inflammatoire élevée et généralisée [2]. Le niveau d'infection avait été défini à partir d'un seuil de CRP $\geq 5 \mathrm{mg} / \mathrm{l}$.

Des mesures anthropométriques (poids, Taille, sexe, âge) de la population étudiée ont complété la collecte des données.

Les mensures anthropométriques des enfants de l'échantillon ont été comparées à celles des enfants de la population de référence ayant les mêmes caractéristiques d'âge, puis des indices nutritionnels ont été calculés. Ainsi, ont été déterminées les différentes formes de malnutrition selon une déviation par rapport à la médiane de - 2 écarts -type ou Z-score. L'analyse des données anthropométriques a été faite à partir du logiciel ENA for SMART muni d'un logiciel EPINUT intégré qui permettait de faire la normalisation des mesures collectées en les comparants à celle de la population de référence de l'OMS de 2006. Ensuite les données ont été transportées sur le logiciel SPSS.17.0 pour les analyses croisées. Le test du $\mathrm{Chi}^{2}$ de Pearson a été utilisé pour la comparaison des moyennes.

Au plan éthique, un consentement préalable était obtenu et les interviews s'étaient déroulées dans le respect de 
l'intimité des ménages. La prise en charge des sujets malades avait été assurée.

\section{Résultats}

La prévalence de l'infection chez les enfants étudiés était significativement plus accentuée en milieu urbain avec 35,2 $\pm 0,47 \%$ qu'en milieu rural avec 14,8 $\pm 0,35$ $\% \quad(p=0,0001)$ (Tableau I). Par contre, elle frappait indifféremment les deux sexes tant en milieu rural qu'urbain sans distinction d'âge. Chez les femmes en âge de procréer étudiées, la prévalence de l'infection était comparable dans les deux milieux avec $24,8 \pm 0,35$ $\%$ en milieu rural et $17,6 \pm 0,47 \%$ en milieu urbain $(p<0,05)$. Aucune différence n'avait été observée entre les classes d'âge aussi bien chez les enfants que les femmes.

L'émaciation et le retard de croissance étaient comparables dans les deux milieux avec respectivement $4,5 \%$ et $28,0 \%$ en milieu rural contre $3,6 \%$ et $6,9 \%$ en zone urbaine (tableau II). Par contre l'insuffisance pondérale était significativement plus importante en milieu rural $(18,4 \%)$ qu'en milieu urbain $(8,9 \%)$ ( $p=$ 0,0001) (Tableau II).

L'association entre retard de croissance et insuffisance pondérale était significative aussi bien en milieu rural qu'urbain (Voir tableau III) avec respectivement $53,3 \%$ et $47,1 \%$ ( $p=0,0001)$. En milieu rural $22 \%$ d'enfants présentant une insuffisance pondérale sont aussi émaciés avec une différence statistiquement significative $(p=0,0001)$. Par contre en milieu urbain, 18,2 \% d'enfants présentaient les deux carences en même temps sans différence significative $(p=0,004)$ (Tableau IV).

La proportion des enfants émaciés et infectés est comparable dans les deux milieux avec respectivement $6,1 \%$ en milieu rural et $4,6 \%$ en milieu urbain. Par contre en milieu rural, la proportion d'enfants ayant un retard de croissance et une insuffisance pondérale étaient nettement plus infectées que ceux du milieu urbain $(p<0,05)$ (tableaux $V$ et $\mathrm{VI})$.

Le déficit énergétique chronique (DEC) en milieu rural était plus important en milieu urbain avec respectivement $13,2 \% \pm 0,65$ et $6,6 \% \pm 0,61$; soit deux fois plus $(p=0,0001)$. II en est de même pour le risque de DEC avec respectivement $24,5 \%$ versus $10,2 \%$. Par contre l'Obésité est plus prononcée en milieu urbain avec 12,7 $\%$ contre seulement $2 \%$ en milieu rural. II en est de même pour le risque d'obésité avec respectivement 26,4 $\%$ contre $6,6 \%$ en milieu rural. ( $p=0,0001$ ). Ainsi, le DEC s'observe plus en milieu rural alors que l'obésité est surtout urbaine.

Le déficit énergétique chronique était comparable selon le milieu de provenance $(p<0,05)$. En milieu rural, l'association infection et déficit énergétique chronique n'était pas significative avec $32,8 \% \pm 0,43$ en milieu rural contre $12,5 \% \pm 0,38$ en milieu urbain.

\section{Discussion}

Déjà en 1996, une étude rétrospective réalisée par FONTAINE $O$ et collaborateurs [7] dans une pouponnière de Côte d'Ivoire concernant 126 enfants malnutris âgés de plus de 15 mois suivis entre 1994 et 1995 avait trouvé que la séroprévalence au $\mathrm{VIH} 1$ était de deux fois supérieure chez les marasmes par rapport aux kwashiorkors (48 \% contre $17 \%$ ). Une autre étude réalisée en 1997 par Niyongabo Theodore sur la malnutrition et l'infection par le $\mathrm{VIH}$ à Paris et à Bujumbura [8] trouve que plus de $33 \%$ de patients sont malnutris, particulièrement au stade de sida, mais parfois dès le stade asymptomatique de l'infection VIH. La même étude observe que l'association entre tuberculose et infection VIH conduit à des situations nutritionnelles graves. L'évaluation de l'impact de différents déterminants sur la survie des patients a montré que la malnutrition est un facteur de mortalité indépendant de l'immunodépression et que le taux de C-réactive protéine constituait également un facteur pronostique indépendant. Des anciens travaux menés en 1984 au Sénégal, avaient permis de constater que les incidences respectives de la diarrhée et de la malnutrition en fonction de l'âge dans les centres de santé étaient pratiquement superposables. Une étude réalisée par OREGA M en milieu pédiatrique en Côte d'Ivoire en 1993 [14] indique que les broncho-pneumopathies représentent $79,55 \%$ des infections pulmonaires chez les enfants malnutris. En 1997 à travers une étude réalisée au CHU de Dakar [9], il est apparu que la diarrhée associée à la malnutrition est l'apanage du nourrisson vivant dans des milieux économiquement défavorisés et surtout lorsque l'état vaccinal n'était pas satisfaisant. Déjà en 1975, Brasseur et al [10] avaient montré à propos d'une étude histologique de la muqueuse intestinale des nourrissons au ZAIRE, que des altérations plus ou moins importantes étaient observées des cas d'émaciation dès l'âge de 4 à 5 mois, et à partir de 20 mois, elles étaient constamment retrouvées chez tous les enfants. Une étude récente menée au Nigeria en 2009 montre que $74.8 \%$ des enfants infectés sont malnutris [13]. Une étude nationale récente de 2006 [3] donnait des résultats d'émaciation plus élevés que ceux de notre étude avec $14 \%$ à Koulikoro contre $4,5 \%$ dans notre étude et $12,4 \%$ à Bamako comparée à $3,6 \%$ pour notre étude. Les résultats pour le retard de croissance et l'insuffisance pondérale étaient comparables. Chez les femmes en âge de procréer, le déficit énergétique chronique était de $13,2 \% \pm 0,65$ en milieu rural contre $6,6 \% \pm 0,61$ en milieu urbain, soit deux fois plus.

\section{Conclusion et recommandations}

Par rapport à l'infection, elle était relativement plus importante en milieu urbain $(35,2 \%$ contre $14,8 \%)$ chez les enfants, alors que chez les femmes, c'est plutôt en milieu rural qu'elle était plus importante avec $24,8 \%$ contre $17,6 \% \quad(p<0,05)$ en milieu urbain. Dans le contexte de situations contraignantes au plan économique et dans le cadre de ressources limitées, la prise en compte simultanée de l'alimentation, des comportements et de l'accès aux soins contribuerait à une meilleure prise en charge des maladies infectieuses. 
REFERENCES BIBLIOGRAPHIQUES

1. KELLY HEDGEPETH A, LLOYD DM, COLVIN A et al. Ethnics differences in C-Reactive Protein concentrations, Clin Chem, 2008 ; 54: (1027-1037).

2. VOLANAKIS JE. Human C-Réactive Protein expression, structure, and human, Mol immunol, $2001 ; 38$ :(189-197).

3. AG IKNANE A. Epidémiologie à la portée de tous, Editions Jamana, $2005: 85 \mathrm{p}$.

4. BRASSEUR H, VIS D. Diarrhée chez l'enfant dans les pays en voie de développement, Revue du Tiers Monde (PARIS) ; 1975, 16 : (567-594)

5. DNSI. Projection de la population à partir du RGPH de 1998 jusqu'en 2012

6. MS. Cellule de planification et de statistique (CPS), Direction Nationale de la Statistique et de l'informatique (DNSI). Enquête Démographique et de Santé, Mali (EDSM III), 2001, Bamako, Juin 2002 : 450 p.

7. FONTAINE O, DIOP B, BEAU JP et al. Diarrhée infantile au Sénégal, Enquête épidémiologique dans un faubourg de Dakar. Méd Trop. Janv-Mars 1984 ; 44, (1) : 27-31.

8. NIYONGABO T. Malnutrition et infection par le VIH à Paris et à Bujumbura, prévalence, mécanismes et signification pronostique, 1997, Paris VI, Thèse doctorat en Médecine, $3 \mathrm{vol} ; 186 \mathrm{p}$.

9. DIOUF S, SARR M, SY H, ABDALLAHI OC, FALL M. Malnutrition et diarrhée chez l'enfant au CHU de Dakar: Aspects cliniques, épidémiologiques et biologiques, Médecine d'Afrique Noire, 1990, 37 (2).

10. MS, Cellule de planification et de statistique (CPS), Direction Nationale de la Statistique et de l'informatique (DNSI), Macro Inc. Enquête Démographique et de Santé Mali,(EDSM IV), 2006, Bamako, Juillet 2007 : $551 \mathrm{p}$.

11. FAO. L'état de l'insécurité alimentaire dans le monde 2000 (FAO, Rome)

12. OMS. Turning the tide of malnutrition: responding to the challenge of the 21st century, Geneva : WHO, 2000 (WHO/NHD/00.7)
13. AMUTA EU, OLUSI TA, HOYMSOU RS: Relationship of intestinal parasitic infections and malnutrition among school children in Makurdi, Benue State - Nigeria. The Internet Journal of Epidemiology. 2009, Volume $7 \mathrm{~N}^{\circ} 1$, $9 p$.

14. OREGA M, OULAI M, CISSE L, NIANGUE-BEUGRE M, SORO-KONE M, MIGAN Y,

M'BENGUE A, PLO J, ANDOH J. Malnutrition et infections pulmonaires dans un service de pédiatrie en milieu tropical; Médecine d'Afrique Noire : 1993, 40 (10).

TABLEAU I: RELATION ENTRE L'EMACIATION ET INFECTION CHEZ LES ENFANTS DE 24 - 59 MOIS

\begin{tabular}{|c|c|c|c|c|}
\hline \multirow[b]{4}{*}{ Emaciation } & \multicolumn{4}{|c|}{ Emaciation $(\mathrm{P} / \mathrm{T}<-2 \mathrm{ET})$} \\
\hline & $\begin{array}{c}\text { Milieu rural } \\
(\%)\end{array}$ & $p$ & $\begin{array}{c}\text { Milieu } \\
\text { urbain } \\
(\%)\end{array}$ & \\
\hline & & & $\mathrm{p}$ & \\
\hline & 4,5 & & 3,6 & \\
\hline Infection & 6,1 & 0,343 & & 0,396 \\
\hline Retard de croissance & 6,6 & 0,174 & 5,9 & 0,479 \\
\hline Insuffisance pondérale & 22,8 & 0,000 & 18,2 & 0,004 \\
\hline
\end{tabular}

TABLEAU II : REPARTITION DU RETARD DE CROISSANCE CHEZ LES ENFANTS DE $24-59 \mathrm{MOIS}$

\begin{tabular}{|lccccc|}
\hline & \multicolumn{2}{c}{$\begin{array}{c}\text { Milieu rural } \\
(I C=0,42)\end{array}$} & \multicolumn{2}{c|}{$\begin{array}{c}\text { Jrbain } \\
(I C=0,25)\end{array}$} & $p$ \\
\cline { 2 - 6 } & Effectif & $\%$ & Effectif & $\%$ & \\
\hline Emaciation & 20 & 4,5 & 9 & 3,6 & $>0,05$ \\
Retard de croissance & 107 & 24,0 & 17 & 6,9 & $>0,05$ \\
Insuffisance pondérale & 82 & 16,4 & 22 & 8,9 & 0,0001 \\
\hline
\end{tabular}

TABLEAU III: RELATION ENTRE RETARD DE CROISSANCE ET INSUFFISANCE PONDERALE CHEZ LES ENFANTS DE 24 - 59 MOIS

\begin{tabular}{|c|c|c|c|c|c|}
\hline & \multicolumn{3}{|c|}{ RETARD DE CROISSANCE } & \multirow{2}{*}{\multicolumn{2}{|c|}{$\begin{array}{llll} & \cdots & p \\
\end{array}$}} \\
\hline & RURALE & & URBAIN & & \\
\hline & Effectif & $\%$ & Effectif & $\%$ & \\
\hline $\begin{array}{l}\text { Insuffisance } \\
\text { pondérale }\end{array}$ & 57 & 53,3 & 8 & 47,1 & 0,0001 \\
\hline Normal et autre & 50 & 46,7 & 9 & 52,9 & \\
\hline Total & 107 & 100,0 & 17 & 100,0 & \\
\hline
\end{tabular}

TABLEAU IV: RELATION ENTRE INSUFFISANCE PONDERALE ET EMACIATION CHEZ LES ENFANTS DE 24 - 59 MOIS

\begin{tabular}{|c|c|c|c|c|c|c|}
\hline & \multicolumn{5}{|c|}{ INSUFFISANCE PONDERALE } & \\
\hline & \multicolumn{3}{|c|}{ RURALE } & \multicolumn{2}{|c|}{ URBAIN } & \\
\hline & Effectif & $\%$ & $p$ & Effectif & $\%$ & $p$ \\
\hline Emaciation & 18 & 22,0 & 0,0001 & 4 & 18,2 & 0,004 \\
\hline Normal et autre & 64 & 78,0 & & 18 & 81,8 & \\
\hline Total & 82 & 100,0 & & 22 & 100,0 & \\
\hline
\end{tabular}

